\title{
The visual system prioritizes locations near corners of surfaces (not just locations near a corner)
}

\author{
Marco Bertamini • Mai Helmy • Daniel Bates
}

Published online: 8 August 2013

(C) Psychonomic Society, Inc. 2013

\begin{abstract}
When a new visual object appears, attention is directed toward it. However, some locations along the outline of the new object may receive more resources, perhaps as a consequence of their relative importance in describing its shape. Evidence suggests that corners receive enhanced processing, relative to the straight edges of an outline (corner enhancement effect). Using a technique similar to that in an original study in which observers had to respond to a probe presented near a contour (Cole et al. in Journal of Experimental Psychology: Human Perception and Performance 27:1356-1368, 2001), we confirmed this effect. When figure-ground relations were manipulated using shaded surfaces (Exps. 1 and 2) and stereograms (Exps. 3 and 4), two novel aspects of the phenomenon emerged: We found no difference between corners perceived as being convex or concave, and we found that the enhancement was stronger when the probe was perceived as being a feature of the surface that the corner belonged to. Therefore, the enhancement is not based on spatial aspects of the regions in the image, but critically depends on figure-ground stratification, supporting the link between the prioritization of corners and the representation of surface layout.
\end{abstract}

Keywords Figure-ground · Convexity · Contour curvature · Corner enhancement effect

It is known that the appearance of a new object captures visual attention (Jonides, 1981; Posner, 1980; Watson \&

\footnotetext{
M. Bertamini $(\bowtie) \cdot$ D. Bates

Department of Psychological Sciences, University of Liverpool, Eleanor Rathbone Building, Liverpool L69 7ZA, UK

e-mail: M.Bertamini@liverpool.ac.uk

M. Helmy

Department of Psychology, Menoufia University,

Shibin El Kom, Egypt
}

Humphreys, 1997), that onsets are more salient than offsets (Cole, Kentridge, Gellatly, \& Heywood, 2003), and that the onset effect is not entirely due to local transients (Cole \& Kuhn, 2009, 2010; Enns, Austen, Di Lollo, Rauschenberger, \& Yantis, 2001). But also there is evidence that not all locations along the outline of the new object attract attention or resources equally. For the human visual system, edge information provides information about boundaries, which in turn are informative about solid shape (Koenderink, 1984; Mach, 1959). With respect to curvature along the contour, regions with high curvatures may be treated differently because they carry more information than do low-curvature regions (Attneave, 1954). Regions with extrema (minima and maxima) of curvature are also informative about part structure (Hoffman \& Richards, 1984). Therefore, testing the relative saliences of different locations along an outline contributes to understanding how object recognition works. The greater salience of corners would support a key role of locations with high curvature in processing shape. Specifically, convex corners may be more salient, in agreement with better performance in tasks in which observers judge relative position (e.g., Bertamini \& Croucher, 2003). Alternatively, concave corners may be more salient. Some evidence for this concavity advantage has been reported (Cohen, Barenholtz, Singh, \& Feldman, 2005), and it has been linked with the role of minima of curvature in part parsing (Hoffman \& Richards, 1984).

In this article, we studied the speed of responding to probes placed near different locations of a simple shape by focusing on the phenomenon known as the corner enhancement effect (Cole, Gellatly, \& Blurton, 2001). Unlike previous studies, we used shading and binocular disparity to create surfaces that formed layers overlapping in depth. Our surfaces appeared more like solid objects than like line drawings, and our manipulations allowed us to test the roles of convexity, concavity, and contour ownership. 


\section{Corner enhancement effect}

Cole et al. (2001) presented a small probe shortly after the presentation of a new object (a simple polygon). The task of the participants was to respond to the probe onset by pressing a key. They found that responses were faster when a probe was presented in a region of space adjacent to a corner of the polygon, relative to a probe presented adjacent to one of the object's straight edges. The effect was replicated in a series of experiments. In one study, responses were faster for corners even when the probe was less likely to appear near a corner than near a straight edge. Therefore, it seems unlikely that observers would adopt a strategy of directing attention to corners on the basis of an explicit expectation that these regions are more important. Furthermore, the effect was also present when the contours were defined by motion and not by luminance. This evidence does not support a low-level explanation based on differential masking at corners.

The last two experiments in Cole et al. (2001) confirmed the corner advantage with a different technique: the temporal order judgment paradigm. Probes near a corner were perceived as appearing earlier in time than did probes near a straight edge. On the basis of the combined evidence from these experiments, Cole et al. (2001) concluded that the region near a corner receives enhanced processing.

In a later article, Cole, Skarratt, and Gellatly (2007) reported six experiments on the corner enhancement effect. They found the following: (a) The effect was stronger for the corners of objects (intrinsic) than for corners that did not define the shape of an object. Specifically, their Experiment 2 included a condition in which the probe appeared next to a corner created by the occlusion of one object by another. Responses in this case were slower than responses to the probe near the corner of object - that is, it was as if the probe was located near a straight edge. (b) Local features (notches) along a triangle's contour had a weaker effect than the corners of the triangle.

(c) The corner effect was absent for probes inside the object.

(d) Enclosing the probe within a secondary object eliminated the corner effect, presumably because the probe did not appear as being directly next to the object. (e) Using an Lshaped object, the corner effect was found for a concave corner (the inside of the L), but this effect was weak as compared to that of convex corners. Cole et al. (2007) argued that the corner enhancement effect is a type of distortion of attention due to the onset of a new object, consistent with other accounts of how new objects distort visual space (Miyauchi, Hikosaka, \& Shimojo, 1992; Ruda, 1998; Suzuki \& Cavanagh, 1997).

The effect has been confirmed by Burnham and Neely (2007) using a visual search task. They reported faster responses for targets located at the intersection of two lines, even for an imaginary intersection that was not actually presented.
They concluded that intersections automatically capture visual-spatial attention.

Figure-ground organization

The corner enhancement effect is an ideal tool to explore how different features of a shape are processed. For instance, more resources may be deployed to locations along a contour with high curvature because they carry more information about shape (Attneave, 1954), or to nonaccidental properties that are useful for object recognition (Biederman, 1987). Some of the available evidence already shows that the effect is affected by object properties. For instance, Cole et al. (2007) reported the absence of enhancement for corners created by occlusion (i.e., non-object-defining corners). Figure-ground organization, therefore, is a critical variable to understand the phenomenon. Corners belong to surfaces, and they are owned by the foreground (unidirectional contour ownership). A change in the figure-ground relation has significant consequences for how shapes are perceived (Koffka, 1935). For instance, a figure-ground reversal, and thus a reversal of ownership, affects visual search (Rensink \& Enns, 1998) and the perception of symmetry (Bertamini, 2010). The importance of contour ownership in perception is also consistent with neurophysiological evidence (Zhou, Friedman, \& von der Heydt, 2000).

Following Cole et al. (2007), in the present work we aimed to assess more directly the role of objectness in the corner enhancement effect. It is known that object representations resulting from figure-ground organization can serve as the basis for visual selection, and therefore that attention can be both space- and object-based (Chen, 2012; Egeth \& Yantis, 1997; Reppa, Schmidt, \& Leek, 2012). One limitation of previous experiments that have examined the corner enhancement phenomenon is that they have used contour outlines. If figure-ground relations are important, outlines are problematic. Although such contours can define an object, surface stratification in these cases is ambiguous. A line can be the occluding edge of a surface, the occluding edge of a hole, or even a thin object, like a wire, that is distinct from both the region outside and the region inside (Bertamini \& Farrant, 2006). Moreover, even assuming that the inside of an object is always perceived as the foreground, it is difficult to compare probes inside and outside the shape while keeping distance from fixation the same. This creates a confound with distance from the geometrical center of the object.

One aspect of the corner enhancement effect is paradoxical. It is known that detection accuracy is better at locations that are the target of a subsequent saccade (Hoffman \& Subramaniam, 1995); it is also known that the natural target of a saccade when a new object appears is its center of area (Melcher \& Kowler, 1999). On this basis, one would predict the opposite of a corner enhancement effect for triangles and 
squares, because the corner is farther away from the center than the straight edge location. However, there is also evidence that saccades may be biased toward more informative locations, such as corners (Bozkov, Bohdaneck, Radil-Weiss, Mitrani, \& Yakimoff, 1982; Vishwanath \& Kowler, 2003), or locations that are more relevant for the task (Guez, Marchal, Gargasson, Grall, \& O'Regan, 1994), in which case they are directed to the center of the local region.

Our experiments did not measure eye movements, and therefore this article cannot resolve these questions. However, comparing performance for the same geometrical shape when it is perceived as either foreground or background allows for a test of the role of figure-ground assignment that is not affected by other geometrical differences between the stimuli.

\section{Foreground advantage}

As a result of figure-ground organization, it is believed that figures have unique properties: They are nearer, they occlude the background, and they own their contours (Palmer, 1999). It is known that target discrimination in the figural region has an advantage over the ground region (Wong \& Weisstein, 1982), that when one region is attended it tends to become figure (Vecera, Flevaris, \& Filapek, 2004), and that what is seen as figure attracts attention. A study on this last aspect needs consideration, because the paradigm used there was the same as that for the corner enhancement effect. Nelson and Palmer (2007) asked participants to respond to a small probe located on either side of a contour. The stimulus was the profile of a face, and therefore the assumption was that convexity and familiarity would make the face appear as foreground. Responses were faster to probes located on the figural side, and this was true for simple response times as well as for target discrimination. One possibility is that features like the nose or the mouth, with high contour curvature, contributed to the effect because contours, and therefore their features, only belong to the foreground. However, the advantage for the figural region (inside condition) was absent for larger distances (2.4 deg from the contour). Cole et al. (2007) also found faster respnses inside the object, but, unlike Nelson and Palmer, they found no effect of distance and no advantage for corners. However, because Cole et al. (2007) did not test beyond $1.1 \mathrm{deg}$ from fixation, a ceiling effect for their inside condition may possibly have masked the corner effect.

In summary, although there is evidence of a figural advantage when responding to a probe, faces (used in both Wong \& Weisstein, 1982, and Nelson \& Palmer, 2007) may be special in attracting attention. It is necessary to test the role of figure-ground organization using manipulations, such as binocular disparity, that make figure-ground relations unambiguous for shapes that are otherwise geometrically identical and unfamiliar.

\section{Corners as convexities}

Another aspect of the corner enhancement effect that needs to be analyzed more closely is convexity. A corner is a singularity along a contour. For smooth contours without discontinuities (or a smoothed version of shapes with corners), peaks of high and low curvature are known as extrema. Both corners and extrema can be either convex or concave, and this difference is important for describing solid shapes. Convexities play a key role for object representation and tend to form parts (Hoffman \& Singh, 1997; Koenderink, 1984; for a review, see Bertamini \& Wagemans, 2013), and some evidence has emerged that responses to convex vertices are faster than responses to concave vertices (Bertamini \& Croucher, 2003; Bertamini \& Mosca, 2004) and that convex deletions are easier to detect than deletions of other singularities (Lamote \& Wagemans, 1999).

If convexities are parts, concavities mark the boundaries between parts (Hoffman \& Richards, 1984). This role of concavities has been used to explain why they are salient in several tasks, including visual search (Hulleman, te Winkel, $\&$ Boselie, 2000) and change detection (Barenholtz, Cohen, Feldman, \& Singh, 2003; but see Bertamini \& Wagemans, 2013, for a critical evaluation of the evidence).

Given the different roles of convexity and concavity in shape description, it is possible that the corner enhancement effect is linked to the perception of parts. If so, a large difference in the sizes of the effect should be present for convex (perceived as parts) versus concave (not perceived as parts) corners.

The L shape used by Cole et al. (2007) was not ideal for comparing convexity and concavity, because of its asymmetry and because it was created with an outline. A better way to compare the two types of vertices would be to reverse figure-ground relations and compare congruent shapes that are perceived as either surfaces or holes (Bertamini, 2006; Bertamini \& Helmy, 2012). The corner enhancement effect can provide a direct test of how the visual system treats convexities and concavities along the contour of an unfamiliar shape.

\section{Summary of experiments}

The stimuli in the present experiments were regions that conveyed the impression of overlapping surfaces. The goal was to extend the effect beyond simple outlines and also to explore whether convex and concave corners are treated equally. To achieve the impression of surfaces that overlapped and in which foreground and background were not ambiguous, we used shading in Experiments 1 and 2 and stereograms in Experiments 3 and 4. The two techniques complement each other. Shading is a powerful cue, but it requires cast shadows located in different positions of the 
stimulus. Random-dot stereograms, on the other hand, take time for binocular fusion, but they have the advantage that surfaces are created on the basis of differences in disparity, and therefore are not visible until after depth relations are processed. This means that there is never any ambiguity about the depth order of surfaces. We aimed to find converging evidence from the two sets of experiments, because this would allow for conclusions about surfaces, independently of how those surfaces were computed by the visual system.

\section{Experiment 1: Figure-ground stratification}

The stimulus was a square region (green) located at the center of a larger round surface (gray). Surface stratification was specified by shading. Therefore, as is illustrated in Figs. 1 and 2, the square region was presented as either a surface casting a shadow on top of the round gray background, or as a hole within the round gray object. Because contour curvature is defined relative to the inside of a surface, the reversal from figure (object) to ground (hole) allows for a comparison between convex and concave corners.

The stimulus was presented on the screen, and $100 \mathrm{~ms}$ later a small line appeared. We created two versions of the experiment, one in which the probe was placed inside the central square, and the other in which the probe was placed outside. These two versions were necessary to test the importance of placing the probe on the surface that the corner belongs to or just next to that surface.

\section{Method}

Participants A group of 22 observers from the University of Liverpool community took part in the study (16 females, six males). The mean age was 24 years. The participants had normal or corrected-to-normal vision, and students received course credits for their time. Eleven took part in the inside version, and eleven in the outside version of the experiment.

Design The factors Objectness (object and hole), Shape of the Probe (horizontal line or vertical line), Vertical Location of the Probe With Respect to the Center of the Screen (high and low), Horizontal Position of the Probe (left and right), and Horizontal Position of the Square (left and right) were factorially combined, for a total of 32 unique stimuli. Each stimulus was presented six times, for a total of 192 trials in a random sequence. Position of the Probe With Respect to the Inside of the Square (inside and outside) was a between-subjects factor. To highlight the size of the corner enhancement effect, and in line with previous studies, we also computed a corner enhancement index. This index was defined as the difference in response times between straight and corner positions.

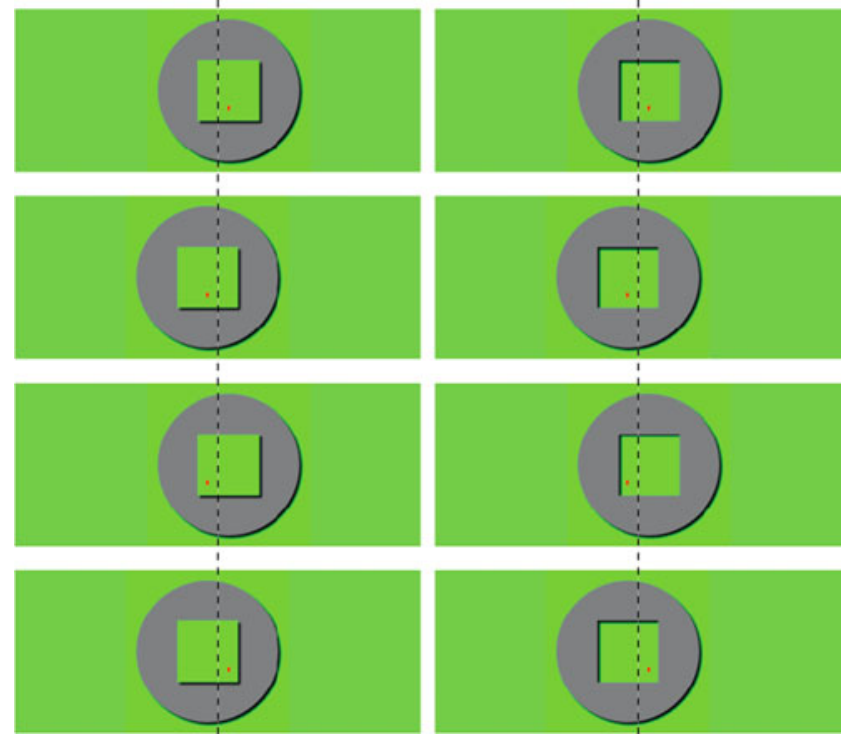

Fig. 1 Sample stimuli for the inside condition of Experiment 1. The stimulus was composed of a long green rectangle (background), a gray surface, and a central green square. In the examples on the left, the square is presented as a surface on top of the gray circle. On the right, the square is a hole within the gray circle. The probe was a small red line that could be vertical (as in these examples) or horizontal (not shown). The top two rows show the probe near the straight part of the contour, because both the square and the probe are shifted horizontally in the same direction relative to the midline, and the bottom two show the probe near a corner, because the square and the probe are shifted in opposite directions. The dashed lines were not part of the display, but are included to show the center of the rectangle, which was also the location of the fixation cross before the stimulus was presented. Note that the probe is always at a fixed distance either to the left or to the right of fixation

Stimuli and procedure The stimuli were presented on a Sony monitor (resolution 1,024 × 768, $85 \mathrm{~Hz}$ ) controlled by a Macintosh computer. The presentation was controlled by a program written in $\mathrm{C}++$ and OpenGL. The fixation mark was presented in the center of the screen on top of a rectangular green background. This background $(200 \times 80 \mathrm{~mm})$ always remained visible (Fig. 3). At the start of a trial, a gray circular surface appeared either slightly to the left or to the right of fixation (distance $5.2 \mathrm{~mm}$ ). In one condition (object), a green square (side $30 \mathrm{~mm}$ ) was centered on the circular gray surface (diameter $70 \mathrm{~mm}$ ). In another condition (hole), the green square was perceived as a hole within the gray surface (see Fig. 1). A probe appeared on the screen $100 \mathrm{~ms}$ after the stimulus. When the horizontal positions of the square and probe were the same, the probe was equidistant from the corners; when they were different, the probe was near a corner (Fig. 1). In both cases, the distance of the probe from fixation was the same. The probe was also displaced vertically by $8.7 \mathrm{~mm}$ either toward the top part of the object or toward the bottom. This factor is referred to as Vertical Location of the Probe (high and low).

Cast shadows provided a sense of depth stratification. They were Gaussian-blurred black regions displaced toward the lower right corner by $14 \mathrm{~mm}$. This corresponds to 


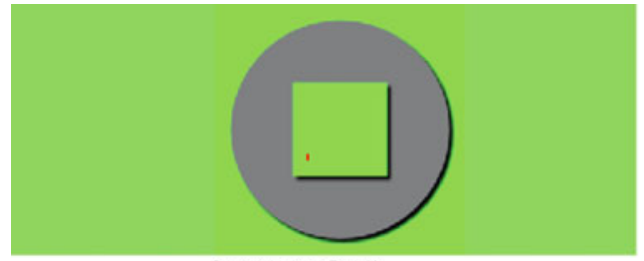

Probe inside (Exp 1)

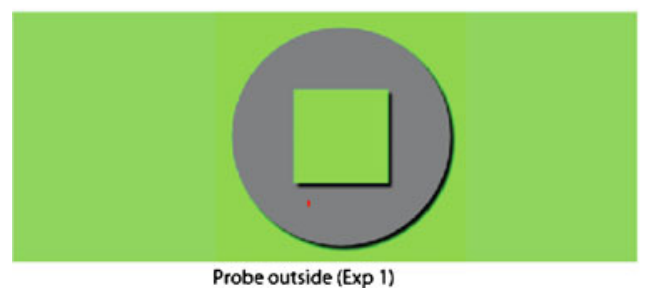

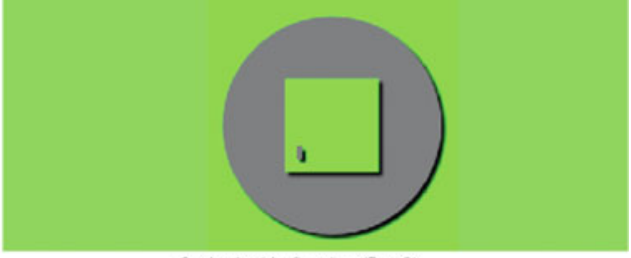

Probe inside floating $(\operatorname{Exp} 2)$

Fig. 2 Examples of stimuli used in Experiment 1 (on the left) and in Experiment 2 (on the right). A small red line was used in Experiment 1, and a gray rectangle with shadow in Experiment 2

lighting from top left, which is the preferred direction for human observers (Mamassian \& Goutcher, 2001). The only source of illumination in the room was a light placed on the left side and higher than the monitor, so that the lighting and direction of shading were consistent.

Observers were seated at approximately $57 \mathrm{~cm}$ from the monitor in a dark and quiet room. We used an eyepatch to ensure that viewing was always monocular, so as to minimize information about the flatness of the monitor. For each participant, we used the dominant eye.

\section{Results and discussion}

Figure 4 shows mean response times. We entered response times in a mixed analysis of variance (ANOVA) with Objectness (object and hole), Corner (corner and straight), and Vertical Location (high and low) as within-subjects factors, and Position (inside and outside) as a between-subjects factor. Errors trials were infrequent $(4.72 \%)$ and were excluded. ${ }^{1}$ Responses more than three $S D$ s from the mean were also excluded $(0.76 \%)$.

Overall, the speeds of responses were not different in the two versions of the experiment [inside and outside, $F(1,20)=$ $0.88, p=.359]$. We observed an effect of corner, with faster responses to probes located near a corner $[F(1,20)=34.49$, $p<$ $\left..001, \eta_{\mathrm{p}}{ }^{2}=0.63\right]$, as well as an interaction between corner and vertical position, with a larger corner advantage for the higher

\footnotetext{
${ }^{1}$ In Experiment 1, the error rates were $4.08 \%$ (straight) and $5.36 \%$ (corner). Given that the task was easy, the error rate was low (on average, nine trials out of 192 per participant in Exp. 1), and likely reflected noise rather than task difficulty. However, for completeness, we transformed errors using the arcsine transformation and performed an ANOVA with the same design as the analysis of response times. No significant effects emerged. These were the steps taken for Experiment 1 , but the same steps and statistical analyses were taken also for the subsequent experiments. In Experiment 2, the error rates were $1.81 \%$ (straight) and $1.71 \%$ (corner). In Experiment 3, the error rates were $1.44 \%$ (straight) and $2.48 \%$ (corner). In Experiment 4, the error rates were $2.82 \%$ (straight) and $3.27 \%$ (corner).
}

position $\left[F(1,20)=7.31, p=.014, \eta_{\mathrm{p}}{ }^{2}=0.26\right]$. A three-way interaction emerged between corner, objectness, and position $\left[F(1,20)=10.25, p=.004, \eta_{\mathrm{p}}{ }^{2}=0.34\right]$, and all other effects were not significant: objectness $[F(1,20)=3.96, p=.060]$, vertical location $[F(1,20)=3.96, p=.465]$, Objectness $\times$ Corner $[F(1,20)=3.96, p=.060]$, and Objectness $\times$ Position $[F(1,20)=1.20, p=.285]$.

To explore the origin of the three-way interaction that emerged from the analysis of response times, we computed the corner enhancement index. In all conditions, this index was positive. A $t$ test compared the effects for objects and for holes, separately for the inside and outside versions of the experiment. In the inside case, the corner advantage was stronger in the object condition $[t(10)=2.36, p<.040$, standardized mean difference $=1.49$ ]. In the outside case, the corner advantage was stronger in the hole condition $[t(10)=2.32, p<.043$, standardized mean difference $=1.46]$. Although these effects are small, it is important to note that they go in opposite directions. This pattern is illustrated in Fig. 5.

These findings show a robust corner enhancement effect, modulated by surface stratification. The effect is stronger for a probe inside the square when the square is perceived as a surface. Conversely, the effect is stronger for a probe outside the square when the square is perceived as an aperture. Therefore, the effect is stronger when the probe is perceived to lie on the surface that the corner belongs to. This is the main finding of Experiment 1. In addition, we can also say that no evidence emerged for a difference in the strengths of the corner enhancement effect between corners perceived as convex or concave. This hypothesis would have been supported by a main effect of objectness, which was not present.

\section{Experiment 2: Probe as an independent surface}

Our interpretation of Experiment 1 relied on the hypothesis that the red probe was perceived as being attached to the 


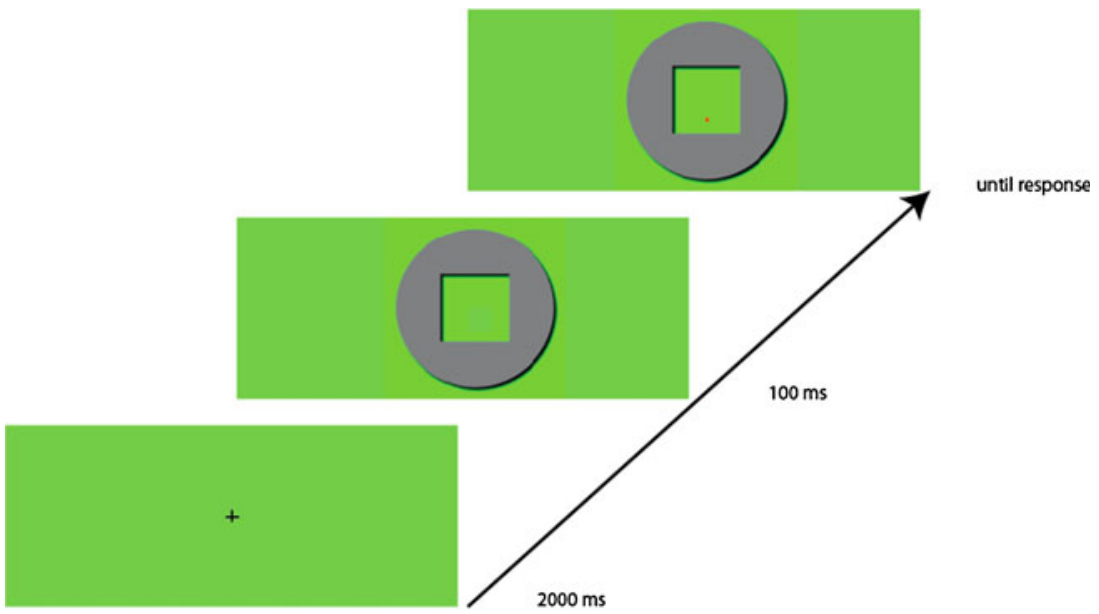

Fig. 3 Procedure of Experiment 1. A fixation cross was presented for 2,000 ms, followed by the onset of a gray object. After another $100 \mathrm{~ms}$ a red probe was presented, and the task was to report whether the probe was horizontal or vertical

surface behind. For a small line this seems reasonable. However, we directly tested this idea in Experiment 2 by using as a probe a small rectangle that was larger than the line and had its own shadow, and therefore appeared to be floating in front of the surface behind. An example is shown in Fig. 2. Because this type of probe is an object that has its own surface and its own depth plane, we predicted a reduction in the corner enhancement effect.
Method

Participants Eleven observers from the University of Liverpool community took part in the study (seven females, four males; mean age 26 years). They had normal or corrected-tonormal vision. Students received course credits for their time.

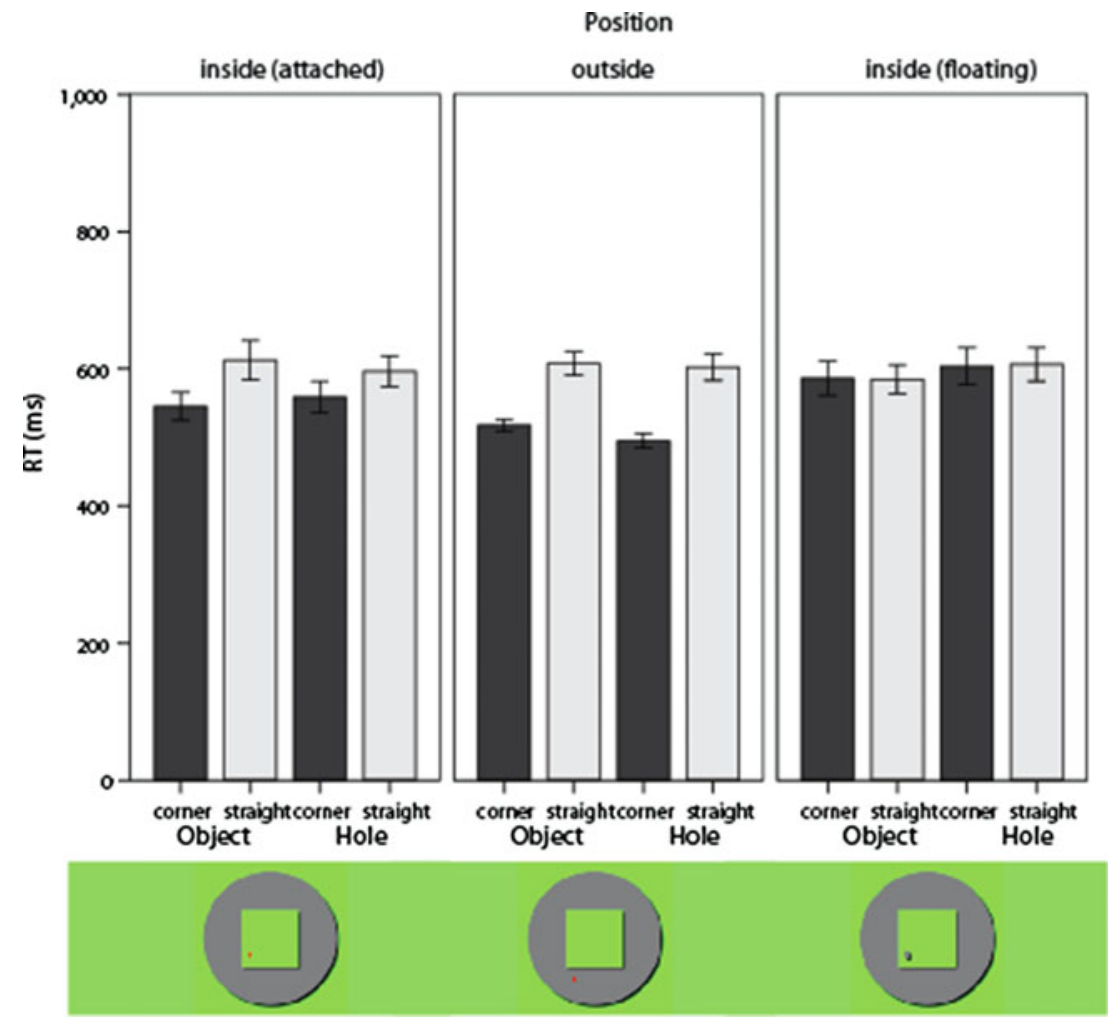

Fig. 4 The first two panels show the results from Experiment 1 (inside and outside positions of the probe). The third panel shows results from Experiment 2 (floating probe). The first and third panels are two types of probes that were present inside the square, and therefore are also labeled "attached" and "floating." Error bars represent 1 SEM 


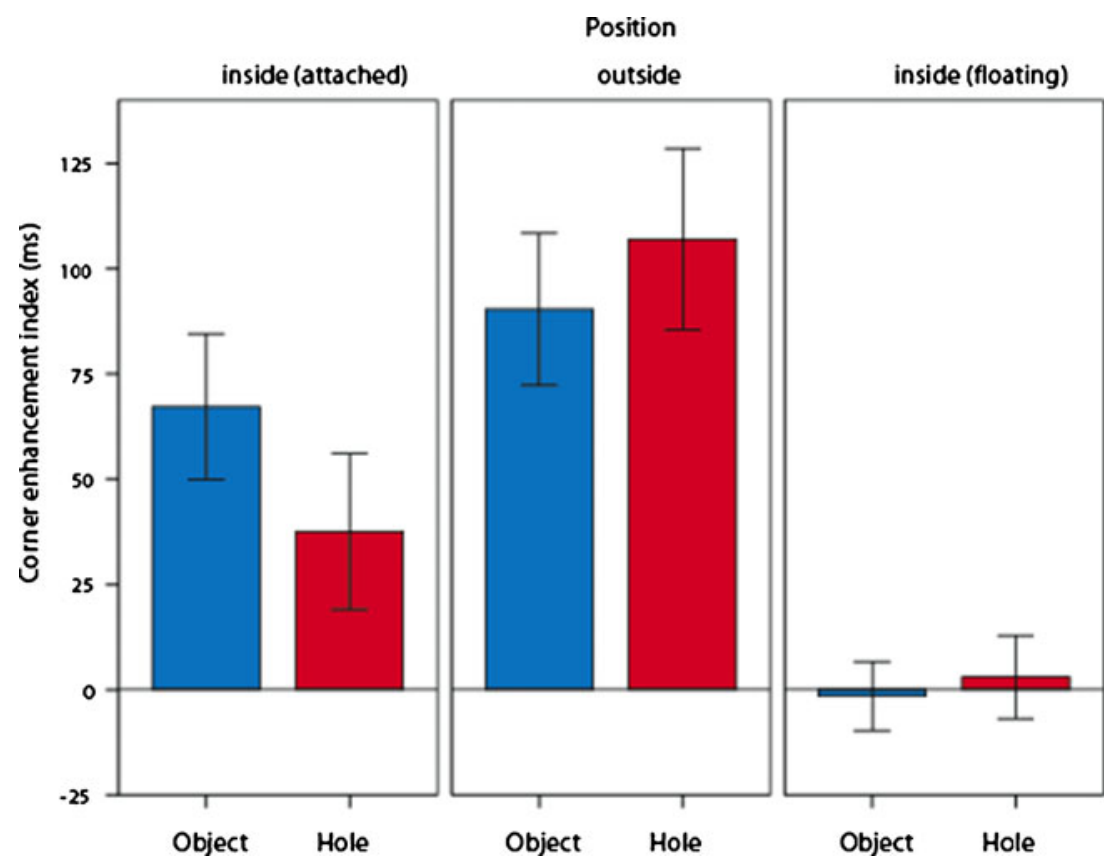

Fig. 5 Sizes of the corner enhancement effect for Experiment 1 (inside and outside positions of the probe) and Experiment 2 (floating probe). The index is the difference between response times in the straight and in the corner conditions. Error bars represent 1 SEM

Design The factors Objectness (object and hole), Shape of the Probe (horizontal line or vertical line), Vertical Location of the Probe With Respect to the Center of the Screen (high and low), Horizontal Position of the Probe (left and right), and Horizontal Position of the Square (left and right) were factorially combined for a total of 32 unique stimuli. Each stimulus was presented six times, for a total of 192 trials in a random sequence. The new data were tested together with the data from the inside condition of Experiment 1, using a mixed ANOVA with Objectness (object and hole), Corner (corner and straight), and Vertical Location (high and low) as within-subjects factors, and Probe (inside line and inside floating rectangle) as a between-subjects factor. Therefore, we now studied two types of inside probes.

Stimuli and procedure Everything was the same as in the inside condition of Experiment 1, except for the appearance of the probe. The new probe was a gray rectangle (height $4.6 \mathrm{~mm}$ ), illustrated in Fig. 2.

\section{Results and discussion}

Figure 4 , right panel, shows mean response times. These data were tested together with the data from the inside condition of Experiment 1. Response times were entered in a mixed ANOVA from which errors trials were excluded. In the version with the line as a probe, the mean error rate was $4.10 \%$, and in the floating-rectangle version, the mean was $1.76 \%$. Responses more than three $S D$ s from the mean were also excluded (1.1\%).
Overall, the speeds of responses were not different in the two versions of the experiment (line and floating rectangle, $F$ $<1.0)$. We found an effect of corner, in which responses were faster to probes located near a corner $[F(1,20)=8.57, p=$ $\left..008, \eta_{\mathrm{p}}{ }^{2}=0.30\right]$. An interaction between corner and probe emerged, in which the corner advantage was larger for the version with a line $\left[F(1,20)=8.017, p=.010, \eta_{\mathrm{p}}{ }^{2}=0.28\right]$. Also, an interaction was apparent between objectness and probe, in which responses were slightly faster for holes in the line version and for objects in the floating-rectangle version $\left[F(1,20)=5.89, p=.025, \eta_{\mathrm{p}}{ }^{2}=0.23\right]$.

Next, the corner advantage index was analyzed as in Experiment 1. In the line probe case, this result was reported earlier: The corner advantage was stronger in the object condition. In the floating-rectangle case, the indices were not different for holes and objects $[t(10)=0.39, p=.704$, standardized mean difference $=0.24]$. As is illustrated in Fig. 5, the corner effect in the floating-rectangle case was not confirmed $[t(10)=0.14, p=.891$, standardized mean difference $=0.08]$. This implies that a critical variable is whether the probe is perceived to be close or attached to the surface that the corner belongs to.

\section{Experiment 3: Random-dot stereograms}

Experiments 1 and 2 confirmed the corner enhancement effect, but the effect was present only when the probe appeared near a corner of a surface, in terms of both location in the image and three-dimensional layout. Depth stratification, 
therefore, is an important factor. Experiments 1 and 2 relied on shading to influence the perceived depth stratification. In Experiment 3, we changed our stimuli so as to present them as stereograms. Disparity was used to specify that the probe was located on the surface on which it appeared. In other words, disparity should support the interpretation of perceived depth that we assume observers perceived in Experiment 1.

The surfaces within the stereograms had the same shape and size as the colored surfaces of Experiments 1 and 2. The use of stereopsis, however, introduces important differences. For instance, disparity information was carried by all dots, and therefore, unlike in the shaded stimuli, depth information was not carried exclusively by some locations (the shadows). Another important difference was that, unlike for monocular cues, the surfaces specified within a random-dot stereogram have no shape until the disparity is processed. The surface stratification, therefore, is unambiguous, as depth itself is what makes the surfaces visible in a random-dot stereogram.

\section{Method}

Participants A group of 22 observers from the University of Liverpool community took part in the study (16 females, six males). The mean age was 20 years, and the participants had normal or corrected-to-normal vision. Before the experiment started, participants' stereo vision was tested with the TNO stereo test. Students received course credits for their time. Eleven took part in the inside version, and eleven in the outside version of the experiment.

Design The design was the same as in Experiment 1. The factors Objectness, Shape of the Probe, Vertical Location of the Probe, Horizontal Position of the Probe, and Horizontal Position of the Square were factorially combined. Position of the Probe With Respect to the Inside of the Square (inside and outside) was a between-subjects factor.

Stimuli and procedure The stimuli were presented on a Sony monitor (resolution $1,280 \times 1,024,120 \mathrm{~Hz}$ ) controlled by a Macintosh computer. The presentation was controlled by a program written in $\mathrm{C}++$ and OpenGL. The stimuli and procedure were identical to those in Experiment 1, except for the following changes. To provide information about depth stratification, we used binocular disparity and random-dot stereograms. Two stereo images were presented with the use of a $\mathrm{NuVision}$ infrared emitter and stereoscopic glasses. Because left and right images were interleaved, the effective vertical resolution and refresh rate were halved $(512$ pixels at $60 \mathrm{~Hz})$. The disparity between the background and the circular surface was 6.9 arcmin, which is not near threshold.

\section{Results and discussion}

Figure 6 shows mean response times. Response times were analyzed in a mixed ANOVA with Objectness (object and hole), Corner (corner and straight), and Vertical Location (high and low) as within-subjects factors, and Position (inside and outside) as a between-subjects factor. Error trials $(1.96 \%)$ and responses that were over three SDs from the mean $(1.4 \%)$ were excluded.

Overall, the speeds of responses were not different in the two versions of the experiment [inside and outside, $F(1,20)=$ $3.09, p=.094]$. We found an effect of objectness, with faster responses to holes than to objects $[F(1,20)=98.30, p<.001$, $\left.\eta_{\mathrm{p}}{ }^{2}=.83\right]$. This may have been due to the greater number of surfaces present in the object condition. An effect of vertical position was also revealed, with faster responses to probes located above fixation $\left[F(1,20)=37.04, p<.001, \eta_{\mathrm{p}}{ }^{2}=.64\right]$.

In terms of significant interactions, we observed interactions between position and vertical position $[F(1,20)=$ 22.85, $\left.p<.001, \eta_{\mathrm{p}}{ }^{2}=.53\right]$, between position and corner $\left[F(1,20)=17.18, p<.001, \eta_{\mathrm{p}}{ }^{2}=.46\right]$, and most importantly, between corner, objectness, and position $[F(1,20)=11.73, p=$ $\left..003, \eta_{\mathrm{p}}{ }^{2}=.37\right]$. Finally, a four-way interaction also emerged $\left[F(1,20)=8.06, p=.010, \eta_{\mathrm{p}}{ }^{2}=.28\right]$.

We used the corner enhancement index to explore the origin of the three-way interaction that emerged from the analysis of response times. In all conditions, this index was positive. We compared the effects for objects and for holes, separately for the inside and outside versions of the experiment. In the inside case, the corner advantage was stronger in the object condition $[t(10)=3.07, p=.012$, standardized mean difference $=1.94]$. In the outside case, no significant difference emerged $[t(10)=1.73, p=.114$, standardized mean difference $=1.09]$. This pattern is illustrated in Fig. 7.

These findings show a robust corner enhancement effect, modulated by surface stratification. The effect is stronger for a probe inside the square when the square is a foreground. The pattern is different, however, when the square is an aperture. In this case, the effect is stronger when the probe is specified to be on the surface that the corner belongs to. This is consistent with the findings of Experiment 1.

One aspect that was different in Experiment 3 than in Experiment 1 was the effect of objectness. Responses were faster for the aperture stimuli than for the object stimuli. Although one could speculate that this was related to concavity (the corners of the aperture) versus convexity (the corners of the foreground), this seems unlikely, given that this effect was absent in Experiment 1. It seems more plausible that the difference was due to the greater amount of disparity information in the object condition: In the case of objects, an additional surface was located on a depth plane that was unoccupied in the aperture condition. 


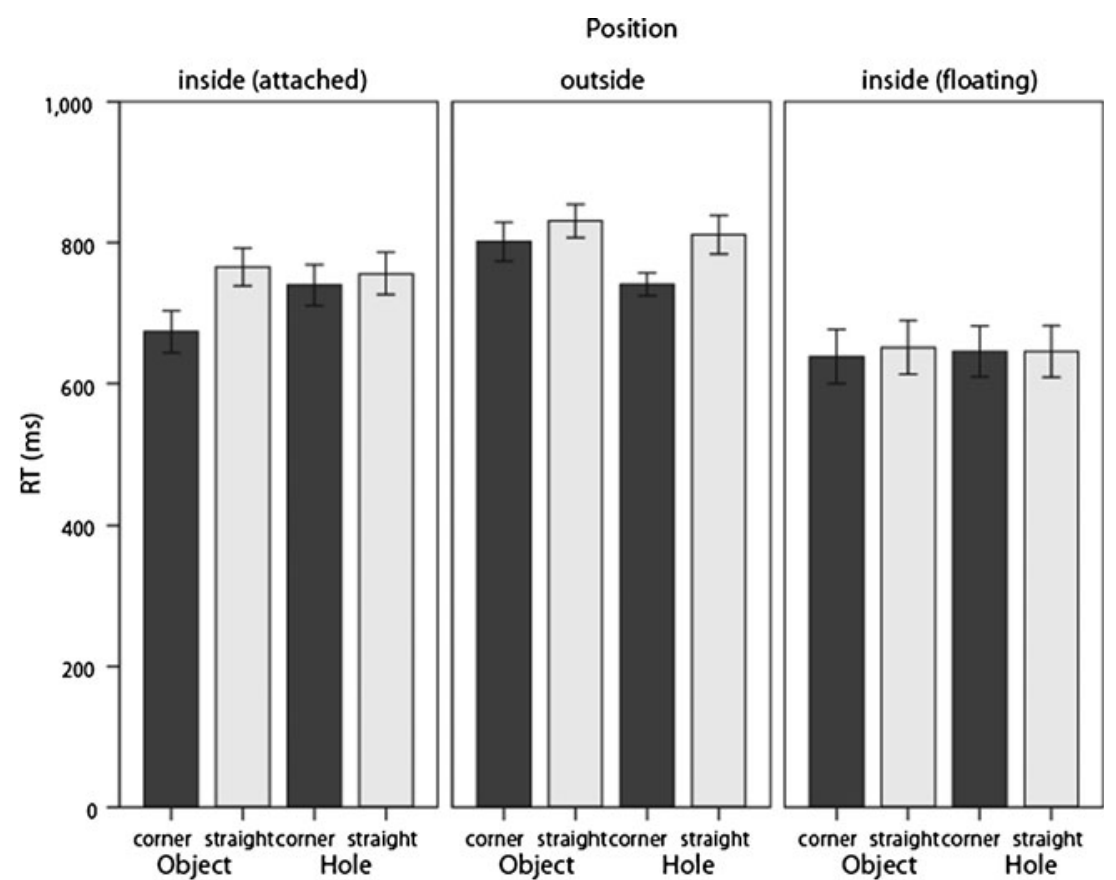

Fig. 6 The first two panels show the results from Experiment 3 (inside and outside positions of the probe). The third panel shows the results from Experiment 4 (floating probe). The first and third panels are two

\section{Experiment 4: Probe at a different depth}

Experiment 3 confirmed the results of Experiment 1: The corner enhancement effect was strongly modulated by depth stratification. In Experiment 2, we had found that a probe perceived as an independent surface did not give rise to a corner enhancement effect. However, one problem with Experiment 2 was that the floating surface was much larger than the small line that had been used in Experiment 1, and also it had its own shadow. Experiment 4 tested the hypothesis that separation in depth is the critical variable and that for a floating probe the enhancement is weak or absent. Using binocular disparity, it was possible to locate the floating vertical bar at a depth that was different from that of the surface behind it, while at the same time retaining the appearance of the probe as being the same as in Experiment 3-that is, a simple line.

\section{Method}

Participants Eleven observers from the University of Liverpool community took part in the study (ten female, one male). The mean age was 21 years, and the participants had normal or corrected-to-normal vision. Before the experiment started, their stereo vision was tested with the TNO stereo test. Students received course credits for their time.

Design The factors Objectness, Shape of the Probe, Vertical Location of the Probe, Horizontal Position of the Probe, and Horizontal Position of the Square were factorially combined. types of probes that were present inside the square, and therefore are also labeled "attached" and "floating." Error bars represent 1 SEM

Response times were analyzed in a mixed ANOVA with Objectness (object and hole), Corner (corner and straight), and Vertical Location (high and low) as within-subjects factors, and Probe (attached and floating) as a betweensubjects factor. This analysis, therefore, directly compared the results from Experiment 4 with the data from the inside condition of Experiment 3, now labeled "attached" in order to contrast them with the floating condition. Therefore, we now studied two types of inside probes.

Stimuli and procedure The stimuli and procedure were identical to those of the inside condition of Experiment 3, with only the exception of the disparity of the line used as probe. The probe in Experiment 4 had a disparity specifying a location nearer the observer. We called this condition floating probe.

\section{Results and discussion}

Figure 6 shows mean response times. Error trials (3.05\%) and responses more than three $S D$ s from the mean $(1.4 \%)$ were excluded.

Overall, the speeds of responses were not different in the two versions of the experiment [attached and floating, $F(1$, $20)=3.71, p=.068]$. An effect of objectness emerged, with faster responses to objects than to holes $[F(1,20)=37.83, p$ $\left.<.001, \eta_{\mathrm{p}}{ }^{2}=.65\right]$. We also found an effect of vertical position, with faster responses to probes located higher 

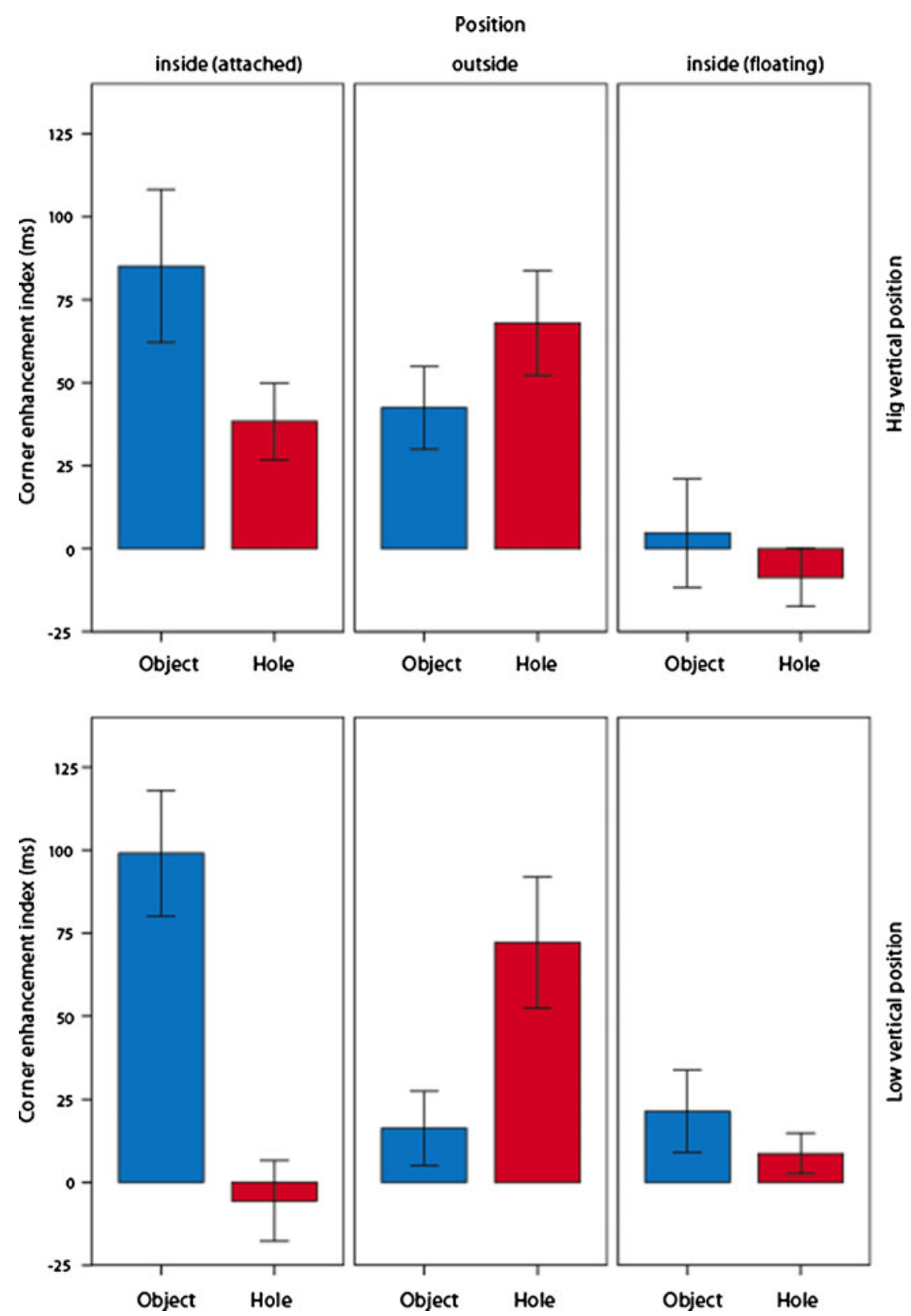

Fig. 7 Sizes of the corner enhancement effect for Experiment 3 (inside and outside positions of the probe) and Experiment 4 (floating probe). The index is the difference between response times in the straight and in

the corner conditions. The two vertical positions (low and high) are shown separately. However, the general pattern is consistent across the two vertical positions. Error bars represent 1 SEM

relative to the center of the square $[F(1,20)=7.52, p=.013$, $\left.\eta_{\mathrm{p}}{ }^{2}=0.27\right]$. In terms of interactions, we observed interactions between objectness and corner $[F(1,20)=12.10, p=$ $\left..002, \eta_{\mathrm{p}}{ }^{2}=.37\right]$ and between objectness and probe $[F(1,20)$ $\left.=23.31, p<.001, \eta_{\mathrm{p}}{ }^{2}=.53\right]$. The most important interaction was the three-way interaction between corner, objectness, and probe $\left[F(1,20)=6.04, p=.023, \eta_{\mathrm{p}}{ }^{2}=.23\right]$.

To explore the three-way interaction, the corner enhancement index was used to compare objects and holes, separately for the attached and floating versions of the experiment. The significant result for the attached case has already been reported (see the inside condition of Exp. 3). In the floating case, no significant difference emerged $[t(10)=1.97, p=$ .077 , standardized mean difference $=1.24]$. This pattern is illustrated in Fig. 7.

The results confirmed that the corner enhancement effect is specific to conditions in which the probe is perceived as being near a corner, but what counted as being "near" was surprising. In the floating-probe condition, the probe was detached from the surface that the corner belonged to, and this may have been the reason that the effect was significantly reduced. However, note that the floating probe was still 
located in the region of the corner, and the difference was due only to depth stratification. This was particularly true in the hole condition, because here the probe was also coplanar with the surface that the corner belonged to. The implication is that the corner enhancement effect requires the probe to appear as a feature of a given surface, and not as an object in a spatial position that is near a corner of an independent surface.

\section{General discussion}

We have studied a recently discovered phenomenon known as the corner enhancement effect (Cole et al., 2001; Cole et al., 2007), in which responses to a probe are faster when the probe appears near a corner of a shape that has appeared together with or slightly before the probe. Cole et al. (2001) established that the phenomenon is robust, extends from simple response times to choice response times, does not depend on the probability of target location, and does not require luminance-defined contours for the object. Cole et al. (2007) also found that the effect does not extend to corners in the image that have been created by occlusion, and they provided some preliminary evidence of the existence of the effect for both convex and concave corners using a large $\mathrm{L}$ shape.

In the introduction, we discussed the phenomenon in the context of the importance of points of high curvature along a contour, in particular convex and concave extrema of curvature. These high-curvature features may play a key role in object recognition (Attneave, 1954; Biederman, 1987) and perceived part structure (Hoffman \& Richards, 1984). We also linked the effect to what is known about attention and figure-ground organization. One can look at the corner enhancement effect as a tool that allows for the probing of any location on or near a visual object and provides a measure of the salience of the feature of the object at that location.

Although there is agreement that information about convexity and concavity along a contour is important, the literature on whether convex and concave regions differ in terms of salience is mixed (for a review, see Bertamini \& Wagemans, 2013). Using an L-shaped object, Cole et al. (2007) found that corner enhancement was present but reduced for a concave corner. By reversing the figure-ground organization for the same region, we were able to test more directly the role of convexity. Using these stimuli, it was possible to locate the probe near the corner of a square perceived as being either foreground (convex corners) or a hole (concave corners). We found that the enhancement effect was not modulated by whether the corner was perceived as convex or concave. Although this may seem surprising, the lack of a difference between convex and concave corners is consistent with the lack of differences between convexity and concavity in change detection (Bertamini, 2008) and in visual search (Bertamini \& Lawson, 2006).

One interesting aspect of the corner enhancement effect is its relation to how attention is directed to a new object, which is also the likely target of a saccade. It is known that before a saccade, attention is shifted toward the saccade target (Deubel \& Schneider, 1996; Kowler, Anderson, Dosher, \& Blaser, 1995) and that the target location for an object tends to be its center of area or center of gravity, especially when the task involves the whole object (He \& Kowler, 1989; Melcher \& Kowler, 1999; Vishwanath \& Kowler, 2003). In our experiments, but also in most experiments conducted by Cole et al. (2001) and by Cole et al. (2007), the center of the object was nearer the straight location than the corner location. However, for extended objects, both attention and saccade locations are also modulated by the task and by the salient features of the object (Guez et al., 1994; Vishwanath \& Kowler, 2003) and by figure-ground relationships (Nelson \& Palmer, 2007).

To further understand how the corner enhancement effect relates to objectness, we used stimuli with a well-defined surface layout. Unlike those in previous studies, our stimuli were perceived as overlapping surfaces, with relative depth information being provided by shading (Exps. 1 and 2) or by binocular disparity (Exps. 3 and 4).

Previous studies had already established that the corner enhancement effect does not require luminance edges (Cole et al., 2001), and can even manifest itself for locations that are implied by a configuration but not actually present in the stimuli (Burnham \& Neely, 2007). However, it was unclear whether the effect had anything to do with objectness, or with salient aspects of a geometric configuration, such as any line intersections. The results using different paradigms were contradictory: Cole et al. (2007) concluded that the effect was absent for nonintrinsic corners (created by overlapping objects), whereas Burnham and Neely concluded that objectness was not important, or at least not necessary.

Our results confirmed the existence of the corner enhancement effect, but not for all configurations. Figures 5 and 7 show the sizes of the effect by plotting the difference in response times between a probe located near a straight edge and a probe located near a corner. We found that the effect was present for both foreground and hole conditions, but it critically depended on the side of the surface on which the probe appeared. The effect was stronger in the object than in the hole condition when the probe appeared inside the square, and therefore on a square region that was perceived as a foreground surface. Conversely, the effect was stronger in the hole than in the object condition when the probe appeared outside the square, and therefore on the foreground surface with a hole.

We suggest that the explanation for this interaction is that the probe needs to be perceived on the foreground because that implies that it appears as being attached to the object that 
the corner belongs to. In other words, the results are consistent with the principle of contour ownership: The effect is stronger in situations in which the probe is perceived near a corner of the object on which it lies, and the effect is weaker in situations in which the probe is perceived to lie on a background surface that extends underneath the corner. One implication of this result is that the corner enhancement effect cannot be explained in terms of the corners present in the image, but depends on surface layout.

This result from Experiment 1 was replicated using random-dot stereograms in Experiment 3. The use of stereograms in which surfaces are only defined by depth stratification showed that the pattern found using luminance-defined contours and shading was replicated for cyclopean contours. As we discussed in the introduction, shading and disparity differ in many respects, and finding converging evidence from both types of stimuli provides generality for our findings.

Experiment 2 introduced a new manipulation of the stimuli: The probe was presented as a distinct object, having its own shadow. Therefore, it was perceived as being detached from the surface behind. This manipulation tested directly the importance of where the probe is perceived, not just in two-dimensional space but also in depth. A floating probe may appear near a corner, and in our hole condition the floating probe was actually coplanar with the corner. Therefore, purely on the basis of three-dimensional distance, one would expect a strong corner enhancement effect. Instead, we failed to see the corner enhancement effect for floating probes. This was true when the percept of floating was achieved with a shadow (Exp. 2) and also with binocular disparity (Exp. 4). We interpreted this as a consequence of the fact that a floating probe has an independent existence, and attention can therefore be directed to the probe independently of the surrounding objects. Conversely, when the probe is not floating, it is perceived as being attached to a surface and is, therefore, a feature of that surface.

In summary, the corner enhancement effect is robust and sensitive to surface layout and depth stratification. We found that the effect is specific to probes that appear to be features of a surface that the corner belongs to. Although we used square regions, these results imply that the corner enhancement effect would be a useful tool to explore attention allocation to more complex shapes, including three-dimensional volumes.

\section{References}

Attneave, F. (1954). Some informational aspects of visual perception. Psychological Review, 61, 183-193. doi:10.1037/h0054663

Barenholtz, E., Cohen, E. H., Feldman, J., \& Singh, M. (2003). Detection of change in shape: An advantage for concavities. Cognition, 89, 1-9.
Bertamini, M. (2006). Who owns the contour of a visual hole? Perception, 35, 883-894. doi:10.1068/p5496

Bertamini, M. (2008). Detection of convexity and concavity in context. Journal of Experimental Psychology: Human Perception and Performance, 34, 775-789. doi:10.1037/0096-1523.34.4.775

Bertamini, M. (2010). Sensitivity to reflection and translation is modulated by objectness. Perception, 39, 27-40.

Bertamini, M., \& Croucher, C. J. (2003). The shape of holes. Cognition, 87(1), 33-54.

Bertamini, M., \& Farrant, T. (2006). The perceived structural shape of thin (wire-like) objects is different from that of silhouettes. Perception, 35, 1679-1692.

Bertamini, M., \& Helmy, M. S. (2012). The shape of a hole and that of the surface-with-hole cannot be analyzed separately. Psychonomic Bulletin \& Review, 19, 608-616. doi:10.3758/s13423-0120265-3

Bertamini, M., \& Lawson, R. (2006). Visual search for a circular region perceived as a figure versus as a hole: Evidence of the importance of part structure. Perception \& Psychophysics, 68, 776-791. doi:10.3758/BF03193701

Bertamini, M., \& Mosca, F. (2004). Early computation of contour curvature and part structure: Evidence from holes. Perception, $33,35-48$

Bertamini, M., \& Wagemans, J. (2013). Processing convexity and concavity along a 2-D contour: Figure-ground, structural shape, and attention. Psychonomic Bulletin \& Review, 20, 191-207. doi:10.3758/s13423-012-0347-2

Biederman, I. (1987). Recognition-by-components: A theory of human image understanding. Psychological Review, 94, 115-147. doi:10. 1037/0033-295X.94.2.115

Bozkov, V., Bohdaneck, Z., Radil-Weiss, T., Mitrani, L., \& Yakimoff, N. (1982). Scanning open and closed polygons. Vision Research, 22, 721-725.

Burnham, B. R., \& Neely, J. H. (2007). Involuntary capture of visualspatial attention occurs for intersections, both real and "imagined. Psychonomic Bulletin \& Review, 14, 735-741. doi:10.3758/ BF03196830

Chen, Z. (2012). Object-based attention: A tutorial review. Attention, Perception, \& Psychophysics, 74, 784-802. doi:10.3758/s13414012-0322-z

Cohen, E. H., Barenholtz, E., Singh, M., \& Feldman, J. (2005). What change detection tells us about the visual representation of shape. Journal of Vision, 5(4):3, 313-321. doi:10.1167/5.4.3

Cole, G. G., Gellatly, A. R. H., \& Blurton, A. (2001). Effect of object onset on the distribution of visual attention. Journal of Experimental Psychology. Human Perception and Performance, 27, 1356-1368.

Cole, G. G., Kentridge, R. W., Gellatly, A. R. H., \& Heywood, C. A. (2003). Detectability of onsets versus offsets in the change detection paradigm. Journal of Vision, 3(1), 3. doi:10.1167/3.1.3

Cole, G. G., \& Kuhn, G. (2009). Appearance matters: Attentional orienting by new objects in the precuing paradigm. Visual Cognition, 17, 755-776

Cole, G. G., \& Kuhn, G. (2010). Attentional capture by object appearance and disappearance. Quarterly Journal of Experimental Psychology, 63, 147-159. doi:10.1080/17470210902853522

Cole, G. G., Skarratt, P. A., \& Gellatly, A. R. H. (2007). Object and spatial representations in the corner enhancement effect. Perception \& Psychophysics, 69, 400-412. doi:10.3758/BF03193761

Deubel, H., \& Schneider, W. X. (1996). Saccade target selection and object recognition: Evidence for a common attentional mechanism. Vision Research, 36, 1827-1837. doi:10.1016/0042-6989(95)00294-4

Egeth, H. E., \& Yantis, S. (1997). Visual attention: Control, representation, and time course. Annual Review of Psychology, 48, 269297. doi:10.1146/annurev.psych.48.1.269

Enns, J. T., Austen, E. L., Di Lollo, V., Rauschenberger, R., \& Yantis, S. (2001). New objects dominate luminance transients in setting 
attentional priority. Journal of Experimental Psychology. Human Perception and Performance, 27, 1287-1302.

Guez, J. E., Marchal, P., Gargasson, J. F. L., Grall, Y., \& O'Regan, J. K. (1994). Eye fixations near corners: Evidence for a centre of gravity calculation based on contrast, rather than luminance or curvature. Vision Research, 34, 1625-1635.

He, P., \& Kowler, E. (1989). The role of location probability in the programming of saccades: Implications for "center-of-gravity" tendencies. Vision Research, 29, 1165-1181.

Hoffman, D. D., \& Richards, W. (1984). Parts of recognition. Cognition, 18, 65-96.

Hoffman, D. D., \& Singh, M. (1997). Salience of visual parts. Cognition, 63, 29-78.

Hoffman, J. E., \& Subramaniam, B. (1995). The role of visual attention in saccadic eye movements. Attention, Perception, \& Psychophysics, 57, 787-795. doi:10.3758/BF03206794

Hulleman, J., te Winkel, W., \& Boselie, F. (2000). Concavities as basic features in visual search: Evidence from search asymmetries. Perception \& Psychophysics, 62, 162-174.

Jonides, J. (1981). Voluntary versus automatic control over the mind's eye's movement. In J. Long \& A. Baddeley (Eds.), Attention and performance $I X$ (pp. 187-203). Hillsdale, NJ: Erlbaum.

Koenderink, J. J. (1984). What does the occluding contour tell us about solid shape? Perception, 13, 321-330.

Koffka, K. (1935). Principles of Gestalt psychology. New York, NY: Harcourt.

Kowler, E., Anderson, E., Dosher, B., \& Blaser, E. (1995). The role of attention in the programming of saccades. Vision Research, 35, 1897-1916. doi:10.1016/0042-6989(94)00279-U

Lamote, C., \& Wagemans, J. (1999). Rapid integration of contour fragments: From simple filling-in to parts-based shape description. Visual Cognition, 6, 345-361.

Mach, E. (1959). The analysis of sensations, and the relation of the physical to the psychical. New York, NY: Dover. Original work published 1897 in German.

Mamassian, P., \& Goutcher, R. (2001). Prior knowledge on the illumination position. Cognition, 81, B1-B9.
Melcher, D., \& Kowler, E. (1999). Shapes, surfaces and saccades. Vision Research, 39, 2929-2946.

Miyauchi, S., Hikosaka, O., \& Shimojo, S. (1992). Visual attention field can be assessed by illusory line motion sensation. Investigative Ophthalmology and Visual Science, 33(Suppl.), 1262.

Nelson, R. A., \& Palmer, S. E. (2007). Familiar shapes attract attention in figure-ground displays. Perception \& Psychophysics, 69, 382392. doi:10.3758/BF03193759

Palmer, S. E. (1999). Vision science: Photons to phenomenology. Cambridge, MA: MIT Press.

Posner, M. I. (1980). Orienting of attention. Quarterly Journal of Experimental Psychology, 32, 3-25. doi:10.1080/00335558008248231

Rensink, R. A., \& Enns, J. T. (1998). Early completion of occluded objects. Vision Research, 38, 2489-2505.

Reppa, I., Schmidt, W. C., \& Leek, E. C. (2012). Successes and failures in producing attentional object-based cueing effects. Attention, Perception, \& Psychophysics, 74, 43-69. doi:10.3758/s13414-011-0211-x

Ruda, H. (1998). The warped geometry of visual space near a line assessed using a hyperacuity displacement task. Spatial Vision, $11,401-419$

Suzuki, S., \& Cavanagh, P. (1997). Focused attention distorts visual space: An attentional repulsion effect. Journal of Experimental Psychology. Human Perception and Performance, 23, 443-463.

Vecera, S. P., Flevaris, A. V., \& Filapek, J. C. (2004). Exogenous spatial attention influences figure-ground assignment. Psychological Science, 15, 20-26. doi:10.1111/j.0963-7214.2004.01501004.x

Vishwanath, D., \& Kowler, E. (2003). Localization of shapes: Eye movements and perception compared. Vision Research, 43, 1637-1653.

Watson, D. G., \& Humphreys, G. W. (1997). Visual marking: Prioritizing selection for new objects by top-down attentional inhibition of old objects. Psychological Review, 104, 90-122. doi:10.1037/ 0033-295X.104.1.90

Wong, E., \& Weisstein, N. (1982). A new perceptual context-superiority effect: Line segments are more visible against a figure than against a ground. Science, 218, 587-589.

Zhou, H., Friedman, H. S., \& von der Heydt, R. (2000). Coding of border ownership in monkey visual cortex. Journal of Neuroscience, 20, 6594-6611. 